\title{
Breast and cervical cancer-screening uptake among females in Ardabil, northwest Iran: a community-based study
}

This article was published in the following Dove Press journal:

OncoTargets and Therapy

17 February 2017

Number of times this article has been viewed

\author{
Esmaeil Farzaneh' \\ Heshmatolah Heydari ${ }^{2}$ \\ Ali Akbar Shekarchi ${ }^{3}$ \\ Aziz Kamran ${ }^{4}$ \\ 'Department of Forensic Medicine \\ and Toxicology, Ardabil University \\ of Medical Sciences, Ardabil, Iran; \\ ${ }^{2}$ Department of Nursing, Lorestan \\ University of Medical Sciences, \\ Khorramabad, Iran; ${ }^{3}$ Department of \\ Pathology, ${ }^{4}$ Department of Public \\ Health, Ardabil University of Medical \\ Sciences, Ardabil, Iran
}

Purpose: Breast and cervical cancers are the most commonly diagnosed type of cancer and cause of cancer-related deaths in Iranian females. In contrast to previous studies, this study was carried out with a large sample size for assessment of breast self-examination (BSE)-, clinical breast examination (CBE)-, mammography-, and Pap smear-uptake rates and determination of associations among these screening behaviors with sociodemographic and cognitive variables in Azeri females.

Materials and methods: This was a cross-sectional, community-based study that was carried out among 1,134 females 20-60 years old during March-June 2016. Data-collection variables included sociodemographic questions, screening behaviors for breast and cervical cancer, self-efficacy, beliefs, and barriers to breast and cervical cancer screening. Collected data were analyzed by SPSS version 13 using $\chi^{2}$, Mann-Whitney $U$, and logistic regression tests.

Results: Among the 1,134 participants, 53.9\%, 9.8\%, and 28.1\% had done BSE, CBE, and Pap smear tests, respectively, and among the 625 females aged $>40$ years, 187 (29.9\%) had done the mammography test. Moreover, 416 (36.7\%), 103 (16.5\%), and 64 (5.6\%) females had done BSE, mammography, and CBE regularly, respectively. Beliefs, barriers, income, health insurance, number of children, and age were all important factors for BSE and regular BSE and mammography. Females who had high belief scores were more likely to undertake mammography (odds ratio [OR]: 1.2, 95\% confidence interval [CI]: 1.03-1.5), regular mammography (OR: 4.2, 95\% CI: 1.9-9.3), regular CBE (OR: 1.25, 95\% CI: 1.2-1.3), and Pap smears (OR: 1.2, 95\% CI: 1.1-1.4). Also, females who had high self-efficacy scores were more likely to perform regular BSE (OR: 1.8, 95\% CI: 1.4-2.5) and mammography (OR: 2.5, 95\% CI: 1.4-4.6) than females with lower self-efficacy scores.

Conclusion: The frequency of breast and cervical cancer screening was low in our study. The findings of this study indicated that beliefs, self-efficacy, and barriers were important predictive factors of cancer-screening behavior among the females studied.

Keywords: breast cancer, cervical cancer, screening, females

\section{Introduction}

Breast cancer is the most commonly diagnosed cancer in females worldwide, and is the leading type and cause of cancer-related deaths in Iranian females too. Breast cancer prevalence is estimated to be $24.6 \%-33.3 \%$ of all cancers in Iran. ${ }^{1-4}$ Recently, evidence has shown that over half a million females will lose their lives as a result of this cancer, ${ }^{2}$ and its incidence is increasing among Iranian females. According to applied research of the Iranian Ministry of Health and Medical Education, during 2006-2010
Correspondence: Aziz Kamran Department of Public Health, Khalkha Faculty of Medical Sciences, Ardabil University of Medical Sciences, Ardabil 56|4995935, Iran Email a.kamran@arums.ac.ir 
in 29 provinces the mortality of breast cancer increased, and there was some evidence to indicate an increase in incidence of this cancer in the future. ${ }^{2}$

Cervical cancer is one of the most preventable cancers and the second-most prevalent cancer among females in the world. ${ }^{5}$ However, the incidence of cervical cancer in Iran is $10 \mathrm{w}^{6}$ and the prevalence of human papillomavirus infection is $7 \%$ among healthy Iranian females, ${ }^{7}$ but the prognosis for cervical cancer is poor and the mortality rate is high: reported as $42 \%-44 \%$ in different studies. ${ }^{5,7}$

Evidence has shown that screening programs for breast cancer could lead to a $20 \%$ reduction in mortality from breast cancer. ${ }^{8}$ Iran's national breast cancer-screening program was introduced in 2012, and recommended that females $>40$ years have an annual mammogram, females 20-40 years old have one clinical breast examination (CBE) every 3 years and annual CBEs after 40 years, and monthly breast self-examination (BSEs) for females $>20$ years. $^{9}$ The Pap smear test is an effective screening program, ${ }^{10}$ and according to the national program cervical cancer screening should be done for all females after marriage, and after three normal Pap smear samples this test should be repeated every 3 years. ${ }^{11}$ This was done free of charge in health care centers, but unfortunately test uptake was not favorable. In a recent Iranian study, $49.4 \%$ of females had done the test once in their lives, ${ }^{10}$ whereas in other countries have reported rates of $85 \%-93 \% .{ }^{12}$ However, the results of previous studies showed a low level of performance and variations in breast cancer screening among Iranian females. ${ }^{13-15}$

Some studies have analyzed uptake for breast and cervical cancer screening among Iranian females, 5,9,13,14 but none of these investigated the cervical and breast cancer screening to include BSE, CBE, mammography, and Pap smears simultaneously. Also, these studies did not examine sociodemographic variables with cognitive variables for determining breast and cervical cancer-screening behaviors. Therefore, this study was carried out to assess BSE, CBE, mammography, and Pap smear screening behaviors among Azeri females (living in Ardabil, northwest Iran) and to study associations among these screening behaviors with demographic and cognitive variables, such as self-efficacy, barriers, and attitudes toward breast and cancer screening.

\section{Materials and methods}

This was a cross-sectional, community-based study conducted in Ardabil city, located in northwest Iran. Ardabil has 238,535 females, and all are Muslims.

\section{Study population}

This study was carried out among 1,134 females 20-60 years old. Participants were recruited from March to June 2016 by trained nursing students. Inclusion criteria were being an Ardabilian female between the ages of 20 and 60 years, without any personal history of breast and cervical cancer, and willingness to participate in the survey. The sampling method was multistage. In the first stage, Ardabil city was divided into three regions according to economic status (low, middle, and high level). Each region was divided into four blocks, and two blocks of each region were randomly selected by simple sampling. In the next stage, public places, such as coffee shops, retail stores, bookstores, childcare facilities, grocery stores, bus stops, and parks, were selected by simple random sampling from each block, and participants were recruited after exploration of the study objectives and expressing their own informed consent. Exclusion criteria were females not consenting to complete the questionnaire and any female with a diagnosis of breast or cervical cancer.

\section{Study procedure}

The data-collection instrument comprised six sections: 1) sociodemographic questions, 2) screening behavior for breast cancer, 3) screening behavior for cervical cancer, 4) self-efficacy, 5) beliefs, and 6) barriers to breast and cervical cancer prevention. The questionnaire was developed based on information obtained from previous studies about breast and cervical cancer screening. Sociodemographic information included age, marital status, level of education, number of children, health insurance coverage, and monthly income. The questionnaire was completed by subjects after introducing the objectives of the study by trained researchers in an appropriate place.

\section{Self-efficacy}

This subscale measured females's confidence in breast and cervical cancer screening by using five items based on a 5 -point Likert scale ( $1=$ strongly disagree, $2=$ disagree, $3=$ no comment, $4=$ agree, $5=$ strongly agree). Those who were scored 5-11 points were grouped in the low level, 12-18 points in the medium level, and 19-25 points in the high level. Example items included: "I am confident that I can schedule and keep a screening test appointment" and "I am confident that I can get a screening test even if I have to pay for it".

\section{Beliefs}

Ten questions were asked on participants' beliefs about breast and cervical cancer prevention. The responses were based 
on the 5-point Likert scale. Example items included: "It is likely that I will get breast or cervical cancer", "Having a screening test is the best way for me to find cancer at an early and curable stage", and "Having a test will decrease my chances of dying from cancer".

\section{Barriers}

Barriers were evaluated by a ten-item scale based on the 5-point Likert scale. Example items included: "I am afraid of having a screening test, because I might find out something is wrong", "Having a screening test is too embarrassing", "Having a screening test takes too much time", "Having a Pap smear or mammogram is too painful", and "I have other problems more important than getting a test". Internal reliability for the self-efficacy, belief, and barrier sections was $0.89,0.84$, and 0.9 , respectively.

Breast cancer screening was assessed using six questions. These questions were: 1) Have you ever had a mammography test (yes/no)?; 2) If yes, regular or irregular?; 3) Have you ever had a BSE (yes/no)?; 4) If yes, regular or irregular?; 5) Have you ever had a CBE (yes/no)?; and 6) If yes, regular or irregular? According to the Iranian health ministry: 1) females 20-40 years old should have a $\mathrm{CBE}$ done every 3 years and annually for high risk females $<30$ years or $>40$ years old; 2 ) for females aged $\geq 40$ years, it is commonly recommended that they have a mammography test every 3 years; and 3 ) females aged $\geq 20$ years should have a BSE monthly. ${ }^{14,16}$

In Iran, cervical cancer screening should be done annually for all females after marriage for 3 years, and after three normal and reliable Pap smear samples, this test should be repeated every 3 years. ${ }^{10}$ Therefore, cervical cancer scoring was assessed by using one question: Have you ever had a Pap smear (yes/no)?

\section{Ethical considerations}

This study was approved by the research-review committee of Khakhal Faculty of Medical Sciences. Verbal informed consent was obtained from all respondents, females participated voluntarily, and the questionnaire was anonymous for quarantine participants' confidentiality.

\section{Data analyses}

Data were analyzed by SPSS version 13 (SPSS Inc, Chicago, IL, USA). Demographic characteristics were analyzed using frequency tables and means. Logistic regression was used to examine associations among self-efficacy, beliefs, barriers, sociodemographic variables, and breast/cervical cancerscreening behaviors. Some of the independent variables were income, insurance, age, marital status, self-efficacy, beliefs, barriers, and education level. The level of significance was $P<0.05$. Odds ratios (ORs) and 95\% confidence intervals (CIs) were determined to explore significant factors associated with cervical and breast cancer-screening behaviors among females who participated in this study.

\section{Results}

Table 1 shows sociodemographic characteristics of the participants in the present study. Among the 1,134 participants, 611 (53.9\%), 111 (9.8\%), and 319 (28.1\%) females had done the BSE, CBE, and Pap smear test, respectively. However, among the 625 aged $>40$ years, 187 (29.9\%) had had a mammography test in their life. Also, results showed that $416(36.7 \%), 103(16.5 \%)$, and 64 (5.6\%) females had undertaken BSE, mammography, and $\mathrm{CBE}$ regularly. The mean age of those who had undertaken BSE and CBE was significantly lower than females who had not. Nevertheless, the mean age of those who had undertaken mammography and Pap smears was significantly higher than the others.

There were significant differences between the two groups (those who had done screening tests and those who had not) with regard to education level, income level, and number of children, but no significant difference was seen for marital status (Table 2). Also, results showed that there were significant differences between females who returned

Table I Sociodemographic characteristics of the participants

\begin{tabular}{lll}
\hline Variables & Frequency & Percentage \\
\hline Age-group & & \\
20-39 years & 509 & 44.9 \\
$40-60$ years & 625 & 55.1 \\
Total & 1,134 & 100 \\
Marital status & & \\
Married & 694 & 61.2 \\
Widow & 132 & 11.6 \\
Separated & 308 & 27.2 \\
Total & 1,134 & 100 \\
Health insurance & & \\
No & 148 & 13.1 \\
Yes & 986 & 86.9 \\
Total & 1,134 & 100 \\
Family income & & \\
Low & 428 & 37.7 \\
Medium & 377 & 33.2 \\
High & 329 & 29 \\
Total & 1,134 & 100 \\
Education level & & \\
Illiterate & 97 & 8.6 \\
Primary school & 268 & 23.6 \\
Middle school & 263 & 23.2 \\
High school & 287 & 25.3 \\
Tertiary & 219 & 19.3 \\
Total & 1,134 & 100 \\
\hline
\end{tabular}




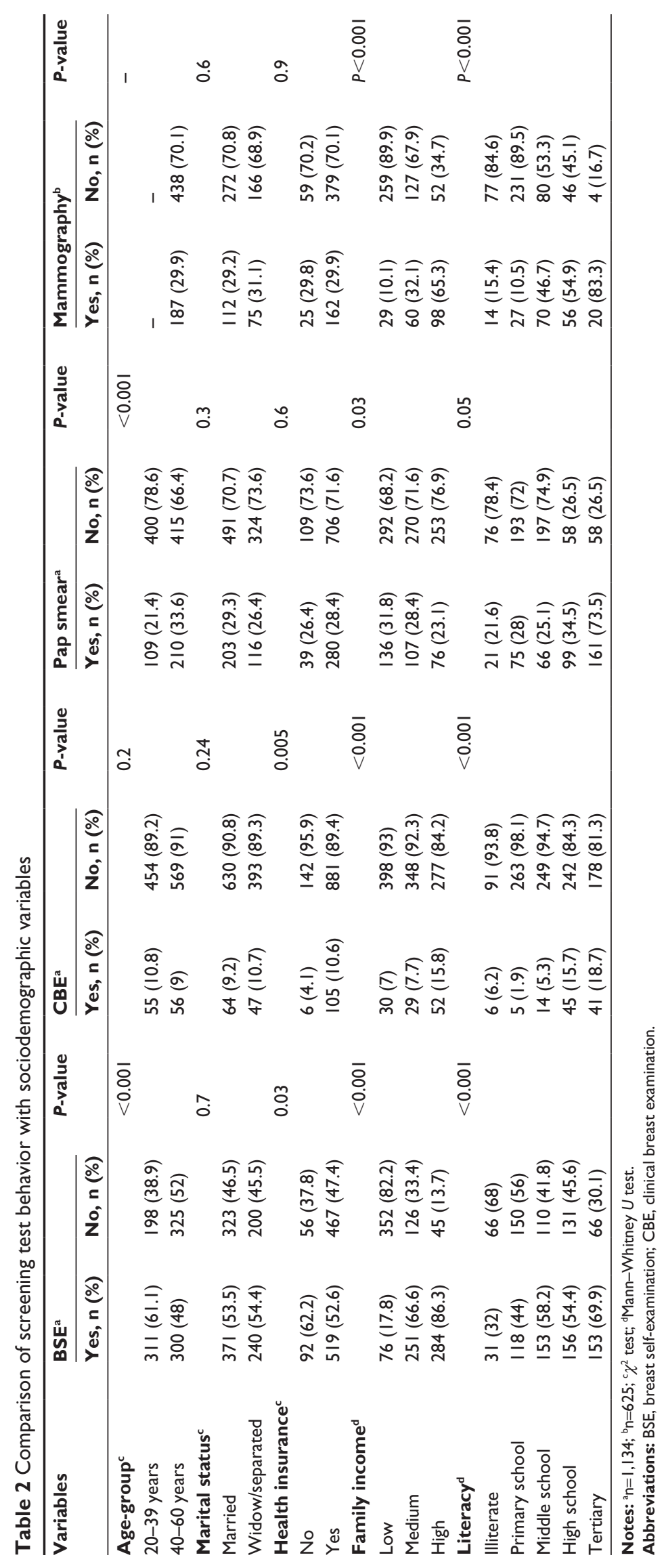


Table 3 Comparison of regular screening test behavior with sociodemographic variables

\begin{tabular}{|c|c|c|c|c|c|c|c|c|c|}
\hline \multirow[t]{2}{*}{ Variables } & \multicolumn{2}{|c|}{ Regular BSE ${ }^{a}$} & \multirow[t]{2}{*}{$P$-value } & \multicolumn{2}{|c|}{ Regular CBE ${ }^{\mathrm{a}}$} & \multirow[t]{2}{*}{$P$-value } & \multicolumn{2}{|c|}{$\begin{array}{l}\text { Regular } \\
\text { mammographyb }\end{array}$} & \multirow[t]{2}{*}{$P$-value } \\
\hline & Yes, n (\%) & No, n (\%) & & Yes, n (\%) & No, n (\%) & & Yes, n (\%) & No, n (\%) & \\
\hline Age-group ${ }^{c}$ & & & 0.03 & & & 0.17 & & & - \\
\hline $20-39$ years & $204(40.1)$ & $305(59.9)$ & & $34(6.7)$ & $475(93.3)$ & & - & - & \\
\hline $40-60$ years & $212(33.9)$ & $413(61.6)$ & & $30(4.8)$ & $595(95.2)$ & & $522(83.5)$ & $103(16.5)$ & \\
\hline Marital status $^{c}$ & & & 0.9 & & & 0.4 & & & 0.7 \\
\hline Married & $255(36.7)$ & $439(63.3)$ & & $36(5.2)$ & $658(94.8)$ & & $65(16.9)$ & $319(83.1)$ & \\
\hline Widow/separated & $161(36.6)$ & $279(63.4)$ & & $28(6.4)$ & $412(93.6)$ & & $38(15.8)$ & $203(84.2)$ & \\
\hline Health insurance ${ }^{c}$ & & & 0.7 & & & 0.1 & & & 0.01 \\
\hline No & $56(37.8)$ & $92(62.2)$ & & $4(2.7)$ & 144 (93.7) & & $6(7.1)$ & $78(92.9)$ & \\
\hline Yes & $360(36.5)$ & $626(63.5)$ & & $60(6.1)$ & $926(93.9)$ & & $97(17.9)$ & $444(82.1)$ & \\
\hline Family income $^{d}$ & & & $<0.00 \mathrm{I}$ & & & 0.002 & & & $<0.001$ \\
\hline Low & $57(13.3)$ & $371(86.7)$ & & $19(4.4)$ & $409(95.6)$ & & $24(8.3)$ & 264 (91.7) & \\
\hline Medium & $107(28.4)$ & $270(71.6)$ & & $14(3.7)$ & $363(96.3)$ & & $40(21.4)$ & 147 (78.6) & \\
\hline High & $252(76.6)$ & $77(23.4)$ & & $31(9.4)$ & $298(90.6)$ & & $39(26)$ & III (74) & \\
\hline Literacy $^{d}$ & & & $<0.00 \mathrm{I}$ & & & $<0.001$ & & & $<0.001$ \\
\hline Illiterate & $19(19.6)$ & $78(80.4)$ & & $2(2.1)$ & 95 (97.9) & & $8(8.8)$ & 83 (9I.2) & \\
\hline Primary school & $76(28.4)$ & $192(71.6)$ & & $2(0.7)$ & $266(99.3)$ & & $10(3.9)$ & $248(96.1)$ & \\
\hline Middle school & $105(39.9)$ & $158(60.1)$ & & $6(2.3)$ & 257 (97.7) & & $40(26.7)$ & $110(73.3)$ & \\
\hline High school & $\mid 20(4 \mid .8)$ & $167(58.2)$ & & $28(9.8)$ & $259(90.2)$ & & $36(35.5)$ & $66(64.7)$ & \\
\hline Tertiary & $96(43.8)$ & $123(56.2)$ & & $26(11.9)$ & $193(88.1)$ & & $9(37.5)$ & $15(62.5)$ & \\
\hline
\end{tabular}

Notes: ${ }^{a} n=1,134$; ${ }^{b} n=625 ;{ }^{c} \chi{ }^{2}$ test; ${ }^{d}$ Mann-Whitney $U$ test.

Abbreviations: BSE, breast self-examination; $\mathrm{CBE}$, clinical breast examination.

for screening tests regularly and females who did not with regard to education level and income level (Table 3).

Beliefs, barriers, income, self-efficacy, number of children, and age were all important factors for prediction of BSE and regular BSE behaviors. Beliefs, barriers, income, selfefficacy, age, and income were important factors for mammography and regular mammography (Table 4). Females who had high belief scores were more likely to undergo mammographies (OR: 1.2, 95\% CI: 1.03-1.5), regular mammographies (OR: 4.2, 95\% CI: 1.9-9.3), regular CBEs (OR: 1.25, 95\% CI: 1.2-1.3), and Pap smears (OR: 1.2, 95\% CI: 1.1-1.4) compared to other females.

Beliefs, barriers, and self-efficacy were important factors for $\mathrm{CBE}$, and beliefs were an important factor for regular CBEs (Table 4). In addition, results showed that beliefs and self-efficacy were important factors in Pap smear screening tests (Table 4). Females with high self-efficacy scores were more likely to perform regular BSEs (OR: 1.8, 95\% CI: 1.4-2.5), regular mammographies (OR: 2.56, 95\% CI: 1.41-4.6) and Pap smears compared to females with low self-efficacy scores (Table 4).

\section{Discussion}

In the present study, the rate of BSE was 53.9\% (611 participants), which was higher than a similar study that reported
49.4\% of Iranian females performing BSEs ${ }^{17}$ and higher than American-Korean females (30.9\% performing BSEs).$^{18}$ This finding is consistent with previous Iranian studies suggesting that the rate of BSE is not satisfactory. ${ }^{19,20}$

The overall uptake of mammography in this study was $29.9 \%$ (187 females), which was higher than that reported among Turkmen females in northeast Iran $(<1 \%)^{13}$ and southeast Iran $(1.5 \%)^{21}$ and lower than a study conducted in Isfahan, a city in central Iran (44.3\%). ${ }^{14}$ This finding revealed the fact that mammography uptake in Iranian females was lower than that reported in developed countries, eg, $93 \%$ in the UK, ${ }^{22} 63.7 \%-84.2 \%$ among American females, ${ }^{23}$ and $64 \%$ among Filipinas. ${ }^{24}$

Results showed that 111 (9.8\%) females had undertaken CBE. This rate was $<53.3 \%$ of Malaysian females aged $20-64$ years who had undertaken $\mathrm{CBE}^{25}$ and contrasted with reported CBE uptake in another Asian study. ${ }^{26}$ The overall uptake of Pap smears in this study was $28.1 \%$ (319 females), which was lower than a reported $91 \%$ of females aged $40-74$ years who had ever had a cervical Pap smear ${ }^{22}$ and lower than Jalilian and Emdadi, in which $63.8 \%$ of participants had undergone Pap smears. ${ }^{27}$ Also, Yu and Rymer reported that $80.5 \%$ of subjects had had a Pap smear atleast once. ${ }^{28}$

In the present study, family income and age were significant factors in BSE, regular BSEs, and mammography 
Table 4 Results of logistic regression analyses

\begin{tabular}{|c|c|c|c|c|c|}
\hline \multirow[t]{2}{*}{ Variables } & \multirow[t]{2}{*}{$P$-value } & \multirow[t]{2}{*}{ OR } & \multicolumn{2}{|l|}{$95 \% \mathrm{Cl}$} & \multirow[t]{2}{*}{$R^{2}$} \\
\hline & & & Lower & Upper & \\
\hline \multicolumn{5}{|l|}{ BSE } & 0.349 \\
\hline Income & $<0.001$ & 5.8 & 4.7 & 7.2 & \\
\hline Beliefs & $<0.001$ & 1.09 & 1.06 & 1.1 & \\
\hline Age & $<0.001$ & 0.98 & 0.96 & 0.99 & \\
\hline \multicolumn{5}{|l|}{ Regular BSE } & 0.388 \\
\hline Income & $<0.001$ & 5.1 & 4.07 & 6.5 & \\
\hline Beliefs & $<0.001$ & I.I & 1.1 & 1.17 & \\
\hline $\begin{array}{l}\text { Number of } \\
\text { children }\end{array}$ & 0.001 & 0.7 & 0.6 & 0.88 & \\
\hline Self efficacy & $<0.001$ & 1.8 & 1.4 & 2.5 & \\
\hline \multicolumn{5}{|c|}{ Mammography (age: 40-60 years) } & 0.68 \\
\hline Income & 0.001 & 8.7 & 2.3 & 24.3 & \\
\hline Beliefs & 0.02 & 1.28 & 1.03 & 1.5 & \\
\hline Barriers & $<0.001$ & 0.28 & 0.16 & 0.48 & \\
\hline Self-efficacy & 0.001 & 1.4 & 1.1 & 1.7 & \\
\hline \multicolumn{5}{|c|}{ Regular mammography (age: $40-60$ years) } & 0.58 \\
\hline Beliefs & $<0.001$ & 4.2 & 1.9 & 9.3 & \\
\hline Self-efficacy & $<0.00$ I & 2.56 & 1.41 & 4.62 & \\
\hline Barriers & 0.006 & 0.16 & 0.04 & 0.6 & \\
\hline Age & 0.026 & 0.51 & 0.29 & 0.9 & \\
\hline \multicolumn{5}{|l|}{ CBE } & 0.373 \\
\hline Beliefs & $<0.001$ & 1.59 & 1.4 & 1.7 & \\
\hline Barriers & 0.005 & 1.5 & 1.3 & 1.7 & \\
\hline \multicolumn{5}{|l|}{ Regular CBE } & 0.22 \\
\hline Beliefs & $<0.001$ & 1.2 & 1.2 & 1.3 & \\
\hline \multicolumn{5}{|l|}{ Pap smear } & 0.21 \\
\hline Self-efficacy & 0.03 & 0.94 & 0.9 & 0.97 & \\
\hline Beliefs & 0.022 & 1.2 & I.I & 1.4 & \\
\hline
\end{tabular}

Abbreviations: BSE, breast self-examination; $\mathrm{CBE}$, clinical breast examination; $\mathrm{OR}$, odds ratio; $\mathrm{Cl}$, confidence interval.

screening behaviors. This finding is in line with other similar studies, ${ }^{22,29-31}$ where the effect of increasing household income was confirmed on the basis of breast and cervical cancer-screening uptake. Moreover, health insurance and education level were significant predictors of BSE, regular BSEs, and mammographies in the present study, which is consistent with similar studies. ${ }^{32-34}$

Other studies have found that females with low incomes had lower participation rates in breast cancer screening, ${ }^{33,35}$ and several studies have shown that higher levels of education were an important predictor for breast and cervical cancer screening. ${ }^{36-38}$ In the present study, family income was an important influencing factor for some cancer-screening test uptake. However, females with higher socioeconomic status were more likely to undergo cancer screening, and these findings were consistent with the results of similar studies. ${ }^{22,39}$ Marital status was not associated with breast/cervical cancerscreening uptake in the present study, but Frie et al found that females who were not married were significantly less likely to attend the screening process. ${ }^{40}$

The results showed that self-efficacy and beliefs were important factors in cancer-screening practices. However, the fact that females with higher self-efficacy scores are more likely to undertake cancer-screening practices is supported by previous research. ${ }^{41-43}$ Hartman et al showed that perceptions of a specific health behavior played an important role in reducing breast cancer risk and engaging in that health behavior. ${ }^{44}$ Consistent with our study, a previous study showed the prediction power of females's confidence in their skills to perform BSE, ${ }^{45}$ and the importance of self-efficacy in mammography and Pap test rates discussed by Kessler ${ }^{46}$ and the significant relationship between beliefs and having Pap smears reported by Jennings-Dozier ${ }^{47}$ are consistent with our study findings. A lack of barriers introduced by Ho et $\mathrm{al}^{48}$ as the most significant predictors of BSE, MBE, and mammography, and also other similar studies, showed the significant relationship between barriers and beliefs with regard to breast cancer examination. ${ }^{42,49}$

\section{Conclusion}

The frequency of performing breast and cervical cancer screening was low in our study. Also, the findings of this study indicated the roles of beliefs, self-efficacy, and barriers were important factors in cancer-screening behavior among the females studied. Therefore, breast and cervical cancerprevention programs should focus on improving self-efficacy and the reduction of barriers to cancer screening.

\section{Limitations}

There were some strengths and limitations for the present study. It was the first study to examine breast (BSE, CBE, and mammography) and cervical (Pap smear) cancer-screening test uptake simultaneously in Iran, especially among Azeri females, who are at a low level economically. However, some other determinants were not studied in the present research, such as family history of cancer and knowledge and awareness of females with regard to breast and cervical cancer.

\section{Disclosure}

The authors report no conflicts of interest in this work.

\section{References}

1. Jazayeri SB, Saadat S, Ramezani R, Kaviani A. Incidence of primary breast cancer in Iran: ten-year national cancer registry data report. Cancer Epidemiol. 2015;39(4):519-527.

2. Enayatrad M, Amoori N, Salehiniya H. Epidemiology and trends in breast cancer mortality in Iran. Iran J Public Health. 2015;44(3):430-431. 
3. Taghavi A, Fazeli Z, Vahedi M, et al. Increased trend of breast cancer mortality in Iran. Asian Pac J Cancer Prev. 2012;13(1):367-370.

4. Silverstein A, Sood R, Costas-Chavarri A. Breast cancer in Africa: limitations and opportunities for application of genomic medicine. Int J Breast Cancer. 2016;2016:4792865.

5. Bahmani A, Baghianimoghadam MH, Enjezab B, Mahmoodabad SS, Askarshahi M. Factors affecting cervical cancer screening behaviors based on the precaution adoption process model: a qualitative study. Glob J Health Sci. 2016;8(6):211-218.

6. Arbyn M, Castellsagué X, de Sanjosé S, et al. Worldwide burden of cervical cancer in 2008. Ann Oncol. 2016;27(9):1-12.

7. Khorasanizadeh F, Hassanloo J, Khaksar N, et al. Epidemiology of cervical cancer and human papilloma virus infection among Iranian women: analyses of national data and systematic review of the literature. Gynecol Oncol. 2013;128(2):277-281.

8. Harding C, Pompei F, Burmistrov D, Welch HG, Abebe R, Wilson R. Breast cancer screening, incidence, and mortality across US counties. JAMA Intern Med. 2015;175(9):1483-1489.

9. Aminisani N, Fattahpour R, Dastgiri S, Asghari-Jafarabadi M, Allahverdipour H. Determinants of breast cancer screening uptake in Kurdish women of Iran. Health Promot Perspect. 2016;6(1):42-46.

10. Farshbaf-Khalili A, Salehi-Pourmehr H, Shahnazi M, Yaghoubi S, Gahremani-Nasab P. Cervical cancer screening in women referred to healthcare centres in Tabriz, Iran. Niger Med J. 2015;56(1):28-34.

11. Farshbaf-Khalili A, Salehi-Pourmehr H, Shahnazi M, Yaghoubi S, Gahremani-Nasab P. [WHO guidelines for screening and treatment of precancerous lesions for cervical cancer prevention]. 2013. Available from: http://apps.who.int/iris/bitstream/10665/94830/5/97892415486 94_per.pdf. Persian.

12. Solomon D, Breen N, McNeel T. Cervical cancer screening rates in the United States and the potential impact of implementation of screening guidelines. CA Cancer J Clin. 2007;57(2):105-111.

13. Charkazi A, Samimi A, Razzaghi K, et al. Adherence to recommended breast cancer screening in Iranian Turkmen women: the role of knowledge and beliefs. ISRN Prev Med. 2013;2013:581027.

14. Moodi M, Rezaeian M, Mostafavi F, Sharifirad GR. Determinants of mammography screening behavior in Iranian women: a populationbased study. J Res Med Sci. 2012;17(8):750-759.

15. Allahverdipour H, Asghari-Jafarabadi M, Emami A. Breast cancer risk perception, benefits of and barriers to mammography adherence among a group of Iranian women. Women Health. 2011;51(3):204-219.

16. Khalili AF, Shahnazi M. Clinical breast exam, and mammography in women referred to health centers in Tabriz, Iran. J Caring Sci. 2012;1(1):37-45.

17. Yadollahie M, Simi A, Habibzadeh F, et al. Knowledge of and attitudes toward breast self-examination in Iranian women: a multi-center study. Asian Pac J Cancer Prev. 2011;12(8):1917-1924.

18. Sadler GR, Ko CM, Cohn JA, White M, Weldon RN, Wu P. Breast cancer knowledge, attitudes, and screening behaviors among African American women: the Black Cosmetologists Promoting Health program. BMC Public Health. 2007;7:57.

19. Jarvandi S, Montazeri A, Harirchi I, Kazemnejad A. Beliefs and behaviours of Iranian teachers toward early detection of breast cancer and breast self-examination. Public Health. 2002;116(4):245-249.

20. Montazeri A, Vahdaninia M, Harirchi I, et al. Breast cancer in Iran: need for greater women awareness of warning signs and effective screening methods. Asia Pac Fam Med. 2008;7(1):6.

21. Heidari Z, Mahmoudzadeh-Sagheb H, Sakhavar N. Breast cancer screening knowledge and practice among women in southeast of Iran Acta Med Iran. 2008;46(4):321-328.

22. Moser K, Patnick J, Beral V. Inequalities in reported use of breast and cervical screening in Great Britain: analysis of cross sectional survey data. BMJ. 2009;338:b2025.

23. Miller JW, King JB, Joseph DA, Richardson LC. Breast cancer screening among adult women: Behavioral Risk Factor Surveillance System, United States, 2010. MMWR Suppl. 2012;61(2):46-50.

24. Wu TY, West B, Chen YW, Hergert C. Health beliefs and practices related to breast cancer screening in Filipino, Chinese and Asian-Indian women. Cancer Detect Prev. 2006;30(1):58-66.
25. Farid ND, Aziz NA, Al-Sadat N, Jamaludin M, Dahlui M. Clinical breast examination as the recommended breast cancer screening modality in a rural community in Malaysia: what are the factors that could enhance its uptake? PLoS One. 2014;9(9):e106469.

26. Norlaili AA, Fatihah MA, Daliana NF, Maznah D. Breast cancer awareness of rural women in Malaysia: is it the same as in the cities? Asian Pac J Cancer Prev. 2013;14(12):7161-7164.

27. Jalilian F, Emdadi S. Factors related to regular undergoing pap-smear test: application of theory of planned behavior. J Res Health Sci. 2011; 11(2):103-108.

28. Yu C, Rymer J. Women's attitudes to and awareness of smear testing and cervical cancer. Br J Fam Plann. 1998;23(4):27-33.

29. Labeit A, Peinemann F. Breast and cervical cancer screening in Great Britain: dynamic interrelated processes. Health Econ Rev. 2015;5(1):32.

30. Challier B, Meslans Y, Viel JF. Deprived areas and attendance to screening of cervix uteri cancer in a French region. Cancer Causes Control. 2000;11(2):157-162.

31. Inumaru LE, Quintanilha MI, da Silveira EA, Naves MM. Risk and protective factors for breast cancer in Midwest of Brazil. $J$ Environ Public Health. 2012;2012:356851.

32. Lairson DR, Chan W, Newmark GR. Determinants of the demand for breast cancer screening among women veterans in the United States. Soc Sci Med. 2005;61(7):1608-1617.

33. Schueler KM, Chu PW, Smith-Bindman R. Factors associated with mammography utilization: a systematic quantitative review of the literature. J Womens Health (Larchmt). 2008;17(9):1477-1498.

34. Tazhibi M, Feizi A. Awareness levels about breast cancer risk factors, early warning signs, and screening and therapeutic approaches among Iranian adult women: a large population-based study using latent class analysis. Biomed Res Int. 2014;2014:306352.

35. Akinyemiju TF. Socio-economic and health access determinants of breast and cervical cancer screening in low-income countries: analysis of the World Health Survey. PLoS One. 2012;7(11):e48834.

36. Damiani G, Federico B, Basso D, et al. Socioeconomic disparities in the uptake of breast and cervical cancer screening in Italy: a cross sectional study. BMC Public Health. 2012;12:99.

37. Sabates R, Feinstein L. The role of education in the uptake of preventative health care: the case of cervical screening in Britain. Soc Sci Med. 2006;62(12):2998-3010.

38. Parise CA, Caggiano V. The influence of socioeconomic status on $\mathrm{racial} /$ ethnic disparities among the ER/PR/HER2 breast cancer subtypes. J Cancer Epidemiol. 2015;2015:813456.

39. Couture MC, Nguyen CT, Alvarado BE, Velasquez LD, Zunzunegui MV. Inequalities in breast and cervical cancer screening among urban Mexican women. Prev Med. 2008;47(5):471-476.

40. Frie KG, Ramadas K, Anju G, et al. Determinants of participation in a breast cancer screening trial in Trivandrum district, India. Asian Pac J Cancer Prev. 2013;14(12):7301-7307.

41. Canbulat N, Uzun O. Health beliefs and breast cancer screening behaviors among female health workers in Turkey. Eur J Oncol Nurs. 2008; 12(2):148-156.

42. Secginli S, Nahcivan NO. Factors associated with breast cancer screening behaviours in a sample of Turkish women: a questionnaire survey. Int J Nurs Stud. 2006;43(2):161-171.

43. Idowu A, Olowookere SA, Fagbemi AT, Ogunlaja OA. Determinants of cervical cancer screening uptake among women in Ilorin, North Central Nigeria: a community-based study. J Cancer Epidemiol. 2016; 2016:6469240

44. Hartman SJ, Dunsiger SI, Jacobsen PB. The relationship of psychosocial factors to mammograms, physical activity, and fruit and vegetable consumption among sisters of breast cancer patients. Int $J$ Womens Health. 2011;3:257-263.

45. Egbert N, Parrott R. Self-efficacy and rural women's performance of breast and cervical cancer detection practices. J Health Commun. 2001; 6(3):219-233.

46. Kessler TA. Increasing mammography and cervical cancer knowledge and screening behaviors with an educational program. Oncol Nurs Forum. 2012;39(1):61-68. 
47. Jennings-Dozier K. Predicting intentions to obtain a Pap smear among African American and Latina women: testing the theory of planned behavior. Nurs Res. 1999;48(4):198-205.

48. Ho V, Yamal JM, Atkinson EN, Basen-Engquist K, Tortolero-Luna G, Follen M. Predictors of breast and cervical screening in Vietnamese women in Harris County, Houston, Texas. Cancer Nurs. 2005;28(2): 119-131.
49. Hasnain M, Menon U, Ferrans CE, Szalacha L. Breast cancer screening practices among first-generation immigrant Muslim women. $J$ Womens Health (Larchmt). 2014;23(7):602-612.

\section{Publish your work in this journal}

OncoTargets and Therapy is an international, peer-reviewed, open access journal focusing on the pathological basis of all cancers, potential targets for therapy and treatment protocols employed to improve the management of cancer patients. The journal also focuses on the impact of management programs and new therapeutic agents and protocols on

\section{Dovepress}

patient perspectives such as quality of life, adherence and satisfaction The manuscript management system is completely online and includes a very quick and fair peer-review system, which is all easy to use. Visit http://www.dovepress.com/testimonials.php to read real quotes from published authors.

\footnotetext{
Submit your manuscript here: http://www.dovepress.com/oncotargets-and-therapy-journal
} 